\title{
What generates flux of tubulin in kinetochore microtubules?
}

by

\author{
Arthur Forer ${ }^{1}$, Jeremy D. Pickett-Heaps² ${ }^{2}$, and Tim Spurck ${ }^{2}$
}

1: corresponding author

address: Biology Department, York University , Toronto, Ontario, Canada M3J 1P3

Email: aforer@yorku.ca

2: Botany Department, University of Melbourne, Parkville, Vic. Australia 3052 


\section{VIEWPOINT}

We reported recently on experiments that probed whether actin and myosin are involved in generating tubulin flux in kinetochore microtubules (kMTs), i.e., in generating the movement of kMT tubulin subunits from kinetochore to pole. We severed kMTs in living metaphase cells using an ultraviolet microbeam, to form a kinetochore stub (KT-stub). Because of continuing tubulin flux, the stub elongated immediately after it was formed: i.e., tubulin is added at the kinetochore as KT-stubs elongate (Maiato et al., 2004; Wilson and Forer, 1989). KT-stub elongation was blocked by the actin inhibitors cytochalasin $\mathrm{D}$ and latrunculin $\mathrm{B}$ and by the myosin inhibitor butanedione monoxime (BDM) (Forer, Spurck and Pickett-Heaps, 2007) so we concluded that actin and myosin facilitate tubulin flux in kMTs. This conclusion is counter to other interpretations that kMT flux is generated solely by microtubules (MTs) and their associated proteins. We now evaluate these differing views.

Flux in spindle MTs has been studied by following small areas of bleached tubulin fluorescence (e.g., Gorbsky et al., 1988), by activation of caged tubulin and following the movement of the spot of fluorescence (e.g., Mitchison, 1989), by following "speckles” (e.g., LaFountain et al., 2004), and by more indirect methods (Wilson et al., 1994; Wilson and Forer, 1997;Forer and Wilson, 2000). Flux in kMTs occurs during both metaphase and in anaphase. Flux during metaphase occurs while kMTs remain of constant length, so tubulin subunits must polymerise at the kinetochore and depolymerise at the pole at equivalent rates. In anaphase the poleward speed of flux is different relative to anaphase chromosome movement in different cell types: flux speeds range from much slower than chromosome movement to considerably faster (review in Rogers et al., 2005), which means that kinetochores sometimes depolymerise kMTs as they move, sometimes are inactive as they move, and sometimes add tubulin subunits to 
kMTs as they move. Thus flux is associated with tubulin depolymerisation at the pole concomitant with tubulin polymerisation, tubulin neutrality, or tubulin depolymerisation at the kinetochore. Various enzymes have been implicated in facilitating the polymerisation and depolymerisation reactions (e.g., Rogers et al., 2004; Ganem et al., 2005; Maiato et al., 2005;Rogers et al., 2005; Civelekoglu-Scholey et al., 2006; Stout et al., 2006). We now look at different models for flux.

Some models consider flux to be driven by depolymerising enzymes at the poles. For example, Rogers et al. (2005) argue that flux is driven by depolymerisation at the poles and that pole-driven flux produces the force to move chromosomes. Recent articles have given theoretical underpinning to the relation between flux and movement. For example, Civelekoglu-Scholey et al. (2006) make a series of assumptions and then solve the force-balance equations numerically, via computer calculations, for ranges of values for the different parameters. Their analysis predicts to a remarkable degree the movements of Drosophila (and other) chromosomes during mitotic metaphase and anaphase (Civelekoglu-Scholey et al., 2006). They assume that the forces that move kinetochores derive from a variety of MT-associated sources: from the action of kMT motors sliding against interpolar MTs, from dynamics of MT elongation and shortening, from MT polymerising enzymes bound to the kinetochore, and from MT depolymerising enzymes at kinetochore and pole. They assume that forces (tension and compression) on the ends of the kMTs that arise from MT forces in various directions drive the enzymatic polymerisation and depolymerisation of kMTs and drive the dynamic reactions of kMTS; and they assume that forces in different directions (including polar ejection forces) together with dynamic reactions inherent in the kMTs give rise to the metaphase equatorial position, to small excursions from the equator, and, after sister chromatid disjunction, to normal anaphase. Their model assumes that 
MT motors (and polymerising and depolymerising enzymes) are the driving forces for flux and for anaphase, and that these are situated solely at the kinetochores and poles (Figure 1A). Results from their modeling match quite well observations of chromosome movements, of equatorial stability or excursions, and of kMT flux seen in living cells, so Civelekoglu-Scholey et al. (2006) explicitly do not consider potential forces generated by interactions of kMTs with components of the spindle matrix.

Our interpretation, however, invokes a spindle matrix (Pickett-Heaps et al. 1984). We have argued previously that forces from a spindle matrix, utilising actin and myosin, are involved both with flux and with chromosome movement. We argued that forces external to the kMTs are applied to kMTs to cause kMT flux and to cause them (and their attached chromosomes) to move poleward during anaphase (Forer and Wilson, 1994; Pickett-Heaps et al., 1996,1997; Spurck et al., 1997; Forer et al., 2003) and that the MTs are rigid struts that limit the rate of movement while directing it spatially. Others have considered that forces from motor proteins attached to a spindle matrix act on kMTs (e.g., Scholey et al., 2001; Johansen and Johansen, 2002; Miyamoto et al., 2004; Mitchison et al., 2005), as in Figure 1B. Our interpretation is different, however, in that we think actin and myosin are involved, and that forces on kMTs may be indirect, via linkages to actin (or myosin), as in Figure 1C. We first discuss evidence for spindle matrix involvement, and then for actin and myosin.

A variety of evidence suggests the spindle matrix is involved in force production in spindles: (1) When a UV microbeam severs newt and PtK fibroblast spindles across the entire half spindle, the poles move closer together; inward pole movements occur in the absence of MT connections across the irradiated region, indicating that there is an underlying tensile element (spindle matrix) that extends throughout the spindle (Spurck et al., 1990; Snyder et al., 1991). (2) 
Crane-fly spermatocyte and newt fibroblast chromosomes can continue to move poleward during anaphase after their kMTs are severed; since there is no MT connection between KT-stub and pole, this indicates that movement is due to external forces acting on the KT-stub (Spurck et al., 1997; Forer et al., 2003). (3) When kMTs are severed, the KT-stubs and the associated chromosomes "wobble" and readjust their positions, as if to re-equilibrate their positions within a tensegral network of forces and MT struts (Spurck et al., 1997; Forer et al., 1997). (4) Newt fibroblast chromosomes accelerate to the poles when KT-stubs are created, as if forces acting on the KT-stubs no longer are hindered by the need to depolymerise intervening kMTs (Spurck et al., 1997). (5) In Xenopus extract spindles treated with a MT depolymerising drug, kMTs buckle as the poles move together, indicating that an underlying "matrix" produces the forces that pull the poles together (Mitchison et al., 2005). (6) Similarly, as newt chromosomes with KT-stubs accelerate poleward, MTs in their path buckle, as if compressed by forces that arise from an underlying matrix (Spurck et al., 1997; Pickett-Heaps et al., 1997). (7) When myosin is hyperphosphorylated by addition of of calyculin, kMTs in metaphase crane-fly spermatocytes buckle (Fabian and Forer, 2007), as if compressed by increased forces from an underlying spindle matrix. (8) When Drosophila S2 cell KT-fibres are severed by a laser microbeam, the chromosomes do not move off the equator and the inter-kinetochore distance does not decrease, implying that forces from the spindle matrix act on the KT-stubs Maiato et al., 2004). (9) Several proteins in Drosophila embryos have properties expected of a "spindle matrix"; for example, they retain the spindle shape even after spindle MTs are depolymerised (e.g., Walker et al., 2000; Rath et al., 2004; Qi et al., 2004; Qi et al., 2005).

Our interpretation that chromosomes move poleward with a KT-stub that has no connection with a pole has been criticised, however. Scholey et al. (2001) suggested that not all 
kMTs were severed ("the efficiency of MT depletion is unknown"), or that kMTs might be linked to adjacent interpolar MTs. Subsequent to that article we showed using confocal and electron microscopy that neither kMTs nor interpolar MTs are present within the irradiated area, that there are no MTs continuous across the irradiated region, and therefore that the chromosomes moved without direct or indirect MT connection to the pole (Forer et al., 2003). Chen and Zhang (2004) also disputed our interpretation. They severed kMTs in grasshopper spermatocytes and reported that the associated chromosomes did not move poleward until the kinetochores captured MTs from the poles, whereupon they moved poleward at speeds 5 times faster than normal before slowing down to normal speeds. However, Chen and Zhang (2004) did not document by confocal or electron microscopy whether MTs near the KT-stubs actually terminated at the kinetochores. Perhaps more importantly, it seems likely to us that, because of the extraordinary speed of these chromosomes, 5 times faster-than-normal, the chromosomes probably moved by sliding along MTs, as during their initial interactions with spindle MTs in other cells (e.g., Tippit et al., 1980; Rieder and Alexander, 1990; Kapoor et al., 2006). Further, Chen and Zhang (2004) suggested that chromosomes with KT-stubs move poleward only when reattached to the pole and do so because of flux driven from depolymerisation at the pole; that seems unlikely to us, since the flux would have had to speed up fivefold during the accelerated movements. For these reasons we are not convinced that our interpretations are invalid, and we continue to suggest that the spindle matrix propels kMTs polewards.

Assuming that the matrix is involved with flux, we argue that actin and myosin are part of the spindle matrix network. Evidence for this includes: (1) Actin and myosin facilitate flux in kMTs, because actin and myosin inhibitors can block flux along non-irradiated kMTs (Silverman-Gavrila and Forer, 2000; Fabian and Forer, 2007) and can block growth of KT-stubs 
produced by ultraviolet microbeam irradiation (Forer et al., 2007). We interpret the latter experiment as in Figure 1D, that matrix-associated motor molecules can continue to act via actin and other non-MT components to propel severed kMTs poleward. (2) Actin and myosin inhibitors can block chromosome attachment to spindle and/or cause detachment of attached chromosomes (e.g., Sampson et al., 1996; Forer and Pickett-Heaps, 1998; Silverman-Gavrila and Forer, 2001). (3) Actin and myosin are present in spindles in a variety of cells, and inhibitors or other anti-myosin or anti-actin treatments often block or otherwise alter anaphase chromosome movements, albeit sometimes only temporarily (summary in Tables I and II of Forer, et al., 2003; also Fabian and Forer, 2005, 2007). (4) Hyper-activation of myosin accelerates anaphase chromosome movements (Fabian et al., 2007). Taken together these findings suggest to us that actin and myosin are intimately involved in chromosome behaviour in mitosis, and, consistent with actin and myosin involvement with MTs in other cell motility systems (e.g., discussed in Fabian and Forer, 2005), we suggest that actin and myosin are components of the spindle matrix that propels kMTs poleward during anaphase (e.g., Fabian, Xia et al., 2007).

Some experiments would seem to counter this interpretation. Mitchison et al. (2005) showed that a spindle matrix produces force on kMTs, but they argued that actin is not involved because the spindles they assemble (from Xenopus egg extracts) are actin-free. This does not necessarily contradict our interpretation, however, because there are redundant mechanisms for force production during anaphase and for flux (e.g., Fabian and Forer, 2005; Cameron et al., 2006). In experiments directly relevant to this issue, for example, Fabian and Forer (2005) showed that even though spindles in non-treated crane-fly spermatocytes can utilise actin (in that actin is associated with the spindle and actin inhibitors can block anaphase chromosome movement), chromosome movement nonetheless is normal in actin-free-spindles (produced by 
continuous immersion in latrunculin from prometaphase onwards). Thus, even though the actinfree spindle matrix can function normally under circumstances when actin has been removed, this does not necessarily mean that actin and myosin are not components of the spindle matrix in vivo.

In sum, we, in common with others, believe that forces and enzyme activities at MT ends are important regulators of MT assembly and disassembly, and believe that various force/flux systems provide redundant mechanisms to move chromosomes. The main difference between our interpretation and other interpretations in the literature is that we consider actin and myosin to be involved (Figure 1). Other interpretations consider the spindle matrix to be involved, but utilising MT motors (e.g., Miyamoto et al., 2004); others that the matrix is not involved (e.g., Rogers et al., 2004; Civelekoglu-Scholey et al., 2006). We think the matrix utilises non-MT motors such as myosin, and perhaps MT motors as well, and that both flux and the chromosome movement system are driven by external forces that act on the kMTs, and are not driven by the kMTs themselves.

\section{ACKNOWLEDGEMENTS}

The work was supported in part by grants from the Natural Sciences and Engineering Research Council (of Canada) to A.F. 


\section{REFERENCES}

Cameron LA, Yang G, Cimini D, Canman JC, Kisurina-Evgenieva O, Khodjakov A, Danuser G, Salmon ED (2006) Kinesin 5-independent poleward flux of kinetochore microtubules in PtK1 cells. J Cell Biol 173: 173-179

Chen W, Zhang D (2004) Kinetochore fibre dynamics outside the context of the spindle during anaphase. Nature Cell Biol 6: 227-231

Civelekoglu-Scholey G, Sharp D J, Mogilner A, Scholey JM (2006) Model of chromosome motility in Drosophila embryos: adaptation of a general mechanism for rapid mitosis. Biophys J 90: 3966-3982

Fabian L, Forer A (2005) Redundant mechanisms for anaphase chromosome movements: cranefly spermatocyte spindles normally use actin filaments but also can function without them. Protoplasma 225: 169-184

Fabian L, Forer A (2007) Possible roles of actin and myosin during anaphase chromosome movements in locust spermatocytes. Protoplasma in press

Fabian L, Troscianczuk J, Forer A (2007) Calyculin A, an enhancer of myosin, speeds up anaphase chromosome movement. BMC Cell and Chromosome 6:1 (17 pages)

Fabian L, Xia A, Venkitaramani DV, Johansen KM, Johansen J, Andrew DJ, Forer A (2007) Titin in insect spermatocyte spindle fibers associates with microtubules, actin, myosin and the matrix proteins skeletor, megator and chromator. J Cell Sci 120: 2190-2204

Forer A, Pickett-Heaps JD (1998) Cytochalasin D and latrunculin affect chromosome behaviour during meiosis in crane-fly spermatocytes. Chromosome Res 6: 533-549

Forer A, Wilson PJ (1994) A model for chromosome movement during mitosis. Protoplasma 179: 95-105 
Forer A, Wilson PJ (2000) Evidence that kinetochore fibre microtubules shorten predominantly at the pole in anaphase flea-beetle spermatocytes. Chromosome Res 8: 151-163

Forer A, Spurck T, Pickett-Heaps JD (1997) Ultraviolet microbeam irradiations of spindle fibres in crane-fly spermatocytes and newt epithelial cells: resolution of previously conflicting observations. Protoplasma 197: 230-240

Forer A, Spurck T, Pickett-Heaps JD, Wilson PJ (2003) Structure of kinetochore fibres in cranefly spermatocytes after irradiation with an ultraviolet microbeam: neither microtubules nor actin filaments remain in the irradiated region. Cell Motil Cytoskeleton 56: 173-192

Forer A, Spurck T, Pickett-Heaps JD (2007) Actin and myosin inhibitors block elongation of kinetochore fibre stubs in metaphase crane-fly spermatocytes. Protoplasma in press

Ganem NJ, Upton K, Compton DA (2005) Efficient mitosis in human cells lacking poleward microtubule flux. Current Biol 15: 1827-1832

Gorbsky GJ, Sammak PJ, Borisy GG (1987) Chromosomes move poleward in anaphase along stationary microtubules that coordinately disassemble from their kinetochore ends. J Cell Biol 104: 9-18

Johansen K M, Johansen J (2002). Recent glimpses of the elusive spindle matrix. Cell Cycle 1: 312-314.

Kapoor TM, Lampson MA, Hergert P, Cameron L, Cimini D, Salmon ED, McEwen BF, Khodjakov A (2006) Chromosomes can congress to the metaphase plate before biorientation. Science 311: 388-391

LaFountain JR Jr, Cohan CS, Siegel AJ, LaFountain DJ (2004) Direct visualization of microtubule flux during metaphase and anaphase in crane fly spermatocytes. Mol Biol Cell 15: 5724-5732 
Maiato H, Rieder CL, Khodjakov A (2004) Kinetochore-driven formation of kinetochore fibers contributes to spindle assembly during animal mitosis. J Cell Biol 167: 831-840

Maiato H, Khodjakov A, Rieder CL (2005) Drosophila CLASP is required for the incorporation of microtubule subunits into fluxing kinetochore fibres. Nature Cell Biol 7: 42-47

Mitchison TJ (1989) Polewards microtubule flux in the mitotic spindle: evidence from photoactivation of fluorescence. J Cell Biol 109: 637-652

Mitchison TJ, Maddox P, Gaetz J, Groen A, Shirasu M, Desai A, Salmon ED, Kapoor TM (2005) Roles of polymerisation dynamics, opposed motors, and a tensile element in governing the length of Xenopus extract meiotic spindles. Molec Biol Cell 16: 3064-3076

Miyamoto, DT, Perlman ZE, Burbank, KS, Groen AC, Mitchison TJ (2004) The kinesin Eg5 drives poleward microtubule flux in Xenopus laevis egg extract spindles J Cell Biol 167: 813-818

Pickett-Heaps J, Spurck T, Tippit D (1984). Chromosome motion and the spindle matrix. J Cell Biol 99: 137s-143s

Pickett-Heaps JD, Forer A, Spurck T (1996) Rethinking anaphase: where "Pac-Man” fails and why a role for the spindle matrix is likely. Protoplasma 192: 1-10

Pickett-Heaps JD, Forer A, Spurck T (1997) Traction fibre: toward a "tensegral” model of the spindle. Cell Motil Cytoskeleton 37: 1-6

Qi H, Rath U, Wang D, Xu Y-Z, Ding Y, Zhang W, Blacketer M, Paddy M, Girton J, Johansen J, Johansen KM (2004) Megator, an essential coiled-coil protein, localizes to the putative spindle matrix during mitosis. Mol Biol Cell 15: 4854-4865 
Qi H, Rath U, Ding Y, Ji Y, Blacketer MJ, Girton J, Johansen J, Johansen KM (2005) EAST interacts with Megator and localizes to the putative spindle matrix during mitosis in Drosophila. J Cell Biochem 95: 1284-1291

Rath U, Wang D, Ding Y, Xu Y-Z, Blacketer MJ, Girton J, Johansen J, Johansen KM (2004) Chromator, a novel and essential chromodomain protein, interacts directly with the spindle matrix protein skeletor in Drosophila. J Cell Biochem 93: 1033-1047

Rieder CL, Alexander SP (1990) Kinetochores are transported poleward along a single astral microtubule during chromosome attachment to the spindle in newt lung cells. J Cell Biol 110: $81-95$

Rodriguez O C, Schaefer AW, Mandato CA, Forscher P, Bement WM, Waterman-Storer CM (2003) Conserved microtubule-actin interactions in cell movement and morphogenesis. Nature Cell Biol 5: 599-609.

Rogers GC, Rogers SL, Schwimmer TA, Ems-McClung SC, Walczak CE, Vale RD, Scholey JM, Sharp DJ (2004) Two mitotic kinesins cooperate to drive sister chromatid separation during anaphase. Nature 427: 364-370

Rogers GC, Rogers SL, Sharp DJ (2005) Spindle microtubules in flux. J Cell Sci 118: 1105-1116 Sampson K, Pickett-Heaps JD, Forer A (1996) Cytochalasin D blocks chromosomal attachment to the spindle in the green alga Oedogonium. Protoplasma 192: 130-144

Scholey JM, Rogers GC, Sharp DJ (2001) Mitosis, microtubules, and the matrix. J Cell Biol 154: 261-266

Silverman-Gavrila RV, Forer A (2000) Evidence that actin and myosin are involved in the poleward flux of tubulin in metaphase kinetochore microtubules of crane-fly spermatocytes. J Cell Sci 113: 597-609 
Silverman-Gavrila RV, Forer A (2001) Effects of anti-myosin drugs on anaphase chromosome movement and cytokinesis in crane-fly primary spermatocytes. Cell Motil Cytoskel 50: $180-197$

Snyder JA, Armstrong L, Stonington OG, Spurck TP, Pickett-Heaps JD (1991) UV-microbeam irradiations of the mitotic spindle: spindle forces and structural analysis of lesions. Eur $\mathrm{J}$ Cell Biol 55: 122-132

Spurck TP, Stonington OG, Snyder JA, Pickett-Heaps JD, Bajer A, Mole-Bajer J (1990) UV microbeam irradiations of the mitotic spindle. II Spindle fiber dynamics and force production. J Cell Biol 111: 1505-1518

Spurck T, Forer A, Pickett-Heaps JD (1997) Ultraviolet microbeam irradiations of epithelial and spermatocyte spindles suggest that forces act on the kinetochore fibre and are not generated by its disassembly. Cell Motil Cytoskeleton 36: 136-148

Stout JR, Rizk RS, Kline SL, Walczak CE (2006) Deciphering protein function during mitosis in PtK cells using RNAi. BMC Cell Biology 7: 26 (15 pages)

Tippit DH, Pickett-Heaps JD, Leslie R (1980) Cell division in two large pennate diatoms Hantzschia and Nitzschia III. A new proposal for kinetochore function during prometaphase. Journal of Cell Biol 86: 402-416

Walker DL, Wang D, Jin Y, Rath U, Wang Y, Johansen J, Johansen KM (2000) Skeletor, a novel chromosomal protein that redistributes during mitosis provides evidence for the formation of a spindle matrix. J Cell Biol 151: 1401-1411

Wilson PJ, Forer A (1989) Identifying the site of microtubule polymerization during regrowth of UV-sheared kinetochore fibres using antibodies against acetylated alpha-tubulin. Cell Biol Int Reports 13: 823-832 
Wilson PJ, Forer A (1997) Effects of nanomolar taxol on crane-fly spermatocyte spindles indicate that acetylation of kinetochore microtubules can be used as a marker of poleward tubulin flux. Cell Motil Cytoskeleton 37: 20-32

Wilson PJ, Forer A, Leggiadro C (1994) Evidence that kinetochore microtubules in crane-fly spermatocytes disassemble during anaphase primarily at the poleward end. J Cell Sci 107: 3015-3027

\section{FIGURE LEGEND}

Figures 1A-C illustrate three different models for producing kMT flux (and force for chromosome movement) and Figure 1D illustrates how the model in C explains the continuation of flux (and force for chromosome movement) after the kMTs are severed. In all illustrations the kinetochore is represented by the front of a boat and the pole by a dock; the kMTs that extend between kinetochore and pole are represented by the thick rope extending between boat and dock; the polymerising and depolymerising enzymes at the kinetochore and dock are represented by winches, the directionality of the winches (represented by arrows in Figure 1A) indicating kMT polymerisation (when the rope is let out), kMT neutrality (when the winch is stationary), or kMT depolymerisation (when the rope is pulled in). Figure $1 A$ represents models in which the driving force for flux and for chromosome movement is depolymerisation of kMTs at the pole: whether the kMTs shorten or elongate depend solely on the action of the winches (enzymes at kinetochore and pole), forces are derived solely from MTs, and depolymerisation at the pole drives flux (and chromosome movement). Figure 1B represents models in which flux depends on motor molecules (represented by the four men) that act on the kMTs. The enzymes at pole and 
kinetochore (winches) remain active, as in illustration A, but the men pulling on the rope drive flux and chromosome movement. That the water can flow, and that the men stand on muddy earth at the bottom of the water, indicates that the matrix might not be stationary and might itself exert force. Figure 1C represents our model in which other motor proteins such as myosin (represented by the three tugboats) act directly on actin filaments (finer wire) which are attached to kMTs (rope) by cross-bridges, thereby indirectly acting on kMTs to propel them poleward and thereby driving flux and chromosome movement. This arrangement of kinetochore fibre actin, myosin and kMTs is not the only arrangement possible, but it is consistent with the staining of actin and myosin in spindles (e.g., Fabian, Xia et al., 2007) and with the cross-linking of actin and microtubules in a variety of other motile systems (e.g., Rodriguez et al., 2003). We do not know enough about the arrangement of actin and myosin with respect to kMTs to evaluate this particular model, but we present it as one possible interpretation of our data, to illustrate the differences in interpretation of the different models. The tugboat "motor molecules" sit on water that can flow in any direction, indicating that the matrix may in itself exert forces. Figure $1 D$ illustrates a KT-stub in which both kMTs and actin are severed by UV microbeam irradiation (Forer et al., 2003), indicating how actin and myosin could facilitate the growth of the KT-stub (Forer et al., 2007) and facilitate continued movement of the chromosome during anaphase (Forer et al., 2003): the tugboats would continue to pull the actin filaments which in turn pull the KT-stub kMTs poleward. The key difference between our interpretation (Figure 1C) and the others (Figures 1A, 1B) is that our interpretation explains how actin/myosin inhibitors block flux and block chromosome movement, and how a myosin enhancer speeds up chromosome movement, whereas the other models do not. [The illustrations are by Gail Rudakewich, http://www.synapse-visuals. com]. 

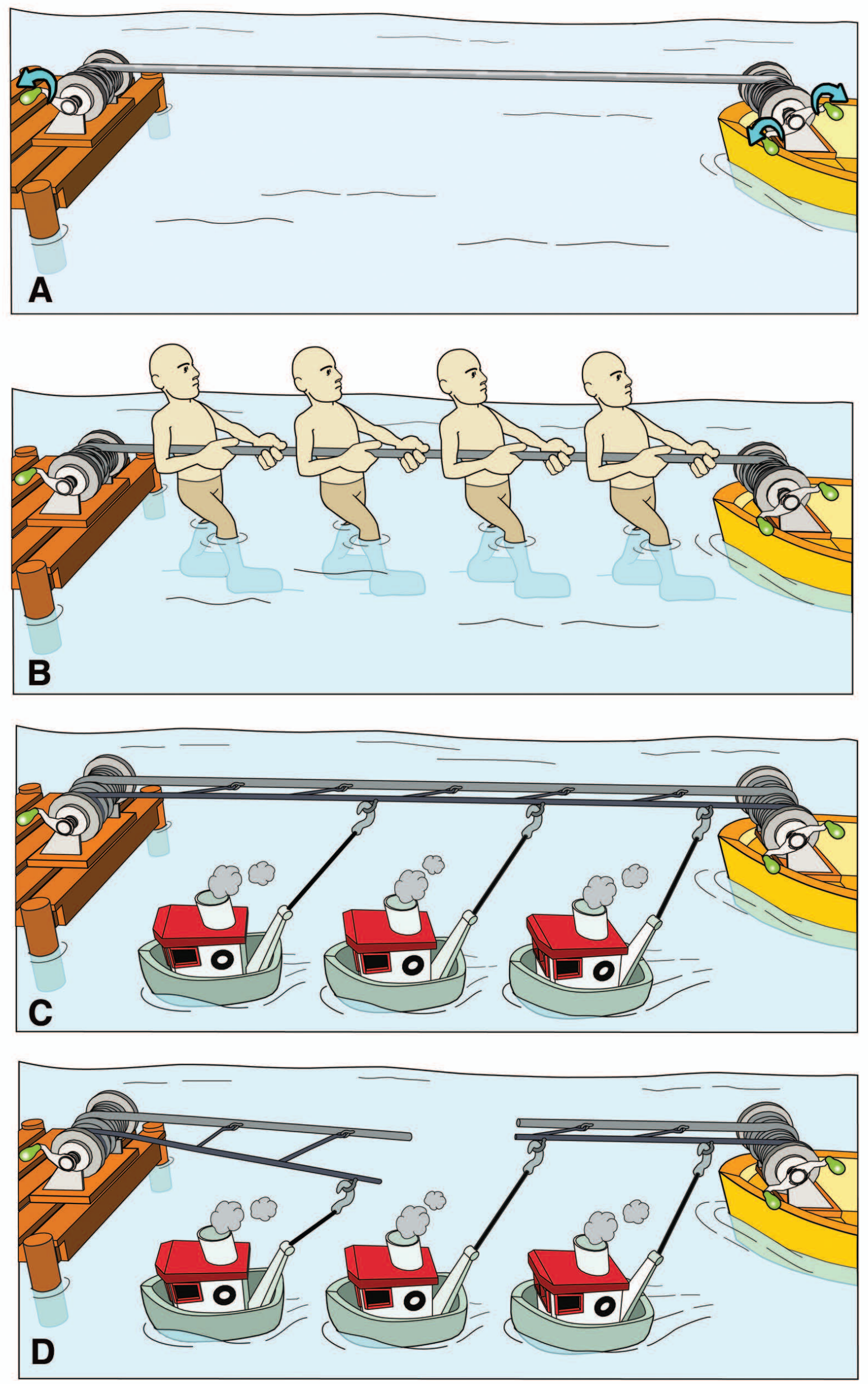